\title{
THE ARITHMETIC OF A SEMIGROUP OF SERIES OF WALSH FUNCTIONS
}

\author{
I. P. IL'INSKAYA
}

(Received 1 July 1998; revised 12 October 1999)

Communicated by. V. Stefanov

\begin{abstract}
Let $\mathscr{W}=\left\{w_{k}(t)\right\}_{k=0}^{\infty}$ be the classical system of the Walsh functions, $\mathscr{S}_{\mathscr{W}}$ the multiplicative semigroup of the functions represented by series of functions $w_{k}(t)$ with non-negative coefficients which sum equals 1 . We study the arithmetic of $\mathscr{S}_{W}$. The analogues of the well-known Khinchin factorization theorems related to the arithmetic of the convolution semigroup of probability measures on the real line are valid in $\mathscr{S}_{W}$. The classes of idempotent elements, of infinitely divisible elements, of elements without indecomposable factors, and of elements without indecomposable and non-degenerate idempotent factors are completely described. We study also the class of indecomposable elements. Our method is based on the following fact: $\mathscr{S}_{W}$ is isomorphic to the semigroup of probability measures on the group of characters of the Cantor-Walsh group.
\end{abstract}

2000 Mathematics subject classification: primary 60B15, 43A25; secondary 42C10.

Keywords and phrases: probability measures on groups, Khinchin factorization theorems, infinite divisibility, indecomposability, Walsh functions.

\section{Introduction and statement of results}

The arithmetic of the convolution semigroup $\mathscr{P}$ of probability measures on $\mathbb{R}^{n}$ has been studied intensively since the 1930 s (see $[9,10]$ ). Nevertheless, some important problems, for example, the problem of the description of the class $I_{0}(\mathscr{P})$ of measures without indecomposable components, remain open. In the 1960s, Kendall, Davidson $[7,8]$, and Urbanik [15] studied semigroups essentially different from $\mathscr{P}$, but with a similar arithmetic. Many other examples of such semigroups were considered later by Bingham, Kennedy, Kingman, Lamperti, Ostrovskii, Ulanovskii (see the expository paper [10]) and by the author $[4,13,14]$. For some of these semigroups, the above mentioned problem has been completely solved.

(C) 2000 Australian Mathematical Society $0263-6115 / 2000 \$ A 2.00+0.00$ 
The aim of this paper is to study a new example of this kind. Let us denote by $r_{i}=r_{i}(t), i=0,1,2, \ldots, t \in[0,1]$, the classical Rademacher functions (see, for example, [5, Chapter 2, Section 2]) defined as

$$
r_{i}(t)=\operatorname{sign}\left(\sin \left(2^{i} \pi t\right)\right)
$$

Let us agree that $r_{i}$ is equal to 1 at the discontinuity points. The Walsh functions are all finite products of Rademacher functions. We set (see [5, Chapter 4, Section 6])

$$
\begin{aligned}
& w_{0}=\psi_{0}^{1}=r_{0} \equiv 1 \\
& w_{1}=\psi_{1}^{1}=r_{1}, \\
& w_{2}=\psi_{2}^{1}=r_{2}, \quad w_{3}=\psi_{2}^{2}=r_{2} r_{1}, \\
& w_{4}=\psi_{3}^{1}=r_{3}, \quad w_{5}=\psi_{3}^{2}=r_{3} r_{1}, \quad w_{6}=\psi_{3}^{3}=r_{3} r_{2}, \quad w_{7}=\psi_{3}^{4}=r_{3} r_{2} r_{1}, \ldots
\end{aligned}
$$

For each $s \in \mathbb{N}$, the Walsh functions of the $s$ th series $\psi_{s}^{j}\left(j=1,2, \ldots, 2^{s-1}\right)$ are products of the function $r_{s}$ and all functions of the preceding series. Let us note that $\psi_{s}^{1}=r_{s}$ for all $s \in \mathbb{N}_{0}:=\mathbb{N} \cup\{0\}$. The Walsh functions form an orthogonal and normalized system on the interval $[0,1]$ with respect to the Lebesgue measure ([5, Chapter 4, Section 5]). Obviously, $r_{k}^{2}(t) \equiv 1, w_{k}^{2}(t) \equiv 1$ for all $k \in \mathbb{N}_{0}$. It is evident that the product of two Walsh functions is a Walsh function as well. The set of all Walsh functions is an abelian group with respect to the multiplication with unity $w_{0}=\psi_{0}^{1} \equiv 1$. Every element of this group is inverse to itself. We introduce the following notation:

$\mathscr{W}:=\left\{w_{k}\right\}_{k=0}^{\infty}$ is the group of the Walsh functions with the discrete topology,

$\mathscr{S}_{\mathscr{W}}$ is the multiplicative semigroup of all functions $f(t), t \in[0,1]$, represented in the form

$$
f(t)=\sum_{k=0}^{\infty} a_{k} w_{k}(t), \quad a_{k} \geq 0, \quad \sum_{k=0}^{\infty} a_{k}=1 .
$$

We endow $\mathscr{S}_{W}$ with the topology of uniform convergence on $[0,1]$.

Our aim is to study the arithmetic of $\mathscr{S}_{\mathscr{W}}$. Let us give the main definitions. The functions $w_{k}, k \in \mathbb{N}_{0}$ are degenerate elements of the semigroup $\mathscr{S}_{W}$. A function $f \in \mathscr{S}_{\mathscr{W}}$ is called idempotent if $f^{2}=f w_{k}$ for some $k \in \mathbb{N}_{0}$. A function $f \in \mathscr{S}_{W}$ is infinitely divisible if for every $n \in \mathbb{N}$ there exist $f_{n} \in \mathscr{S}_{\mathscr{W}}$ and $k \in \mathbb{N}_{0}$ such that $f=\left(f_{n}\right)^{n} w_{k}$. A function $f_{1} \in \mathscr{S}_{\mathscr{W}}$ is called a factor of $f \in \mathscr{S}_{\mathscr{W}}$ if there exists $f_{2} \in \mathscr{S}_{W}$ such that $f=f_{1} f_{2}$. A function $f \in \mathscr{S}_{\mathscr{W}}$ is called indecomposable if $f \neq w_{k}$ for all $k \in \mathbb{N}_{0}$ and if all factors of $f$ are of the forms $w_{k}$ and $f w_{k}$ only. We introduce the following notation:

$\operatorname{Id}\left(\mathscr{S}_{\mathscr{W}}\right)$ is the class of all idempotent functions of $\mathscr{S}_{\mathscr{W}}$;

$I\left(\mathscr{S}_{\mathscr{W}}\right)$ is the class of all infinitely divisible functions of $\mathscr{S}_{\mathscr{W}}$; 
$I_{0}\left(\mathscr{S}_{W}\right)$ is the class of functions without indecomposable and non-degenerate idempotent factors;

$\tilde{I}_{0}\left(\mathscr{S}_{W}\right)$ is the class of functions without indecomposable factors;

$N\left(\mathscr{S}_{W}\right)$ is the class of indecomposable functions.

In Section 2 we show that $\mathscr{S}_{W}$ is isomorphic to the semigroup of probability measures on the group of the characters of the Cantor-Walsh group. Therefore we may use results about the factorization of the probability measures on locally compact abelian groups. From the results of Parthasarathy, Rao and Varadhan $[11,12]$ it follows that three theorems given below are valid in $\mathscr{S}_{W}$.

THEOREM 1 ([12]). Every function $f \in \mathscr{S}_{\mathscr{W}}$ can be represented in the form $f=$ $f_{1} f_{2} f_{3}$, where $f_{1}$ is the maximal idempotent factor of $f, f_{2} \in I_{0}\left(\mathscr{S}_{W}\right), f_{3}$ is a product of the empty, finite or countable set of indecomposable functions (in the first case $f_{3} \equiv 1$, and in the third case the infinite product converges uniformly on $\left.[0,1]\right)$.

THEOREM 2 ([12, 2, Corollary 4.7]). $\tilde{I}_{0}\left(\mathscr{S}_{W}\right) \subset I\left(\mathscr{S}_{W}\right)$.

Theorem 1 and Theorem 2 are analogues of the Khinchin theorems [9, pages 79, 88 ], related to the arithmetic of the semigroup $\mathscr{P}$.

THEOREM 3 ([11]). The class $N\left(\mathscr{S}_{\mathscr{W}}\right)$ is a dense $G_{\delta}$-set in $\mathscr{S}_{\mathscr{W}}$ with respect to the topology of uniform convergence on $[0,1]$.

The main results of our paper are characterizations of the classes $I d\left(\mathscr{S}_{W}\right), I\left(\mathscr{S}_{W}\right)$, $\tilde{I}_{0}\left(\mathscr{S}_{W}\right), I_{0}\left(\mathscr{S}_{W}\right)$ and a test for the membership of $N\left(\mathscr{S}_{W}\right)$.

THEOREM 4. The class Id( $\left.\mathscr{S}_{W}\right)$ consists of all functions $f$ representable in the form

$$
f=w_{j} \prod_{w_{i} \in K}\left(0.5+0.5 w_{i}\right)
$$

where $j \in \mathbb{N}_{0}$ and $K$ is an arbitrary finite subgroup of $\mathscr{W}$.

THEOREM 5. The class $I\left(\mathscr{S}_{\mathbb{W}}\right)$ consists of all functions $f$ representable in the form

$$
f=f_{1} \exp \left(\sum_{k=1}^{\infty} c_{k}\left(w_{k}-1\right)\right),
$$

where $c_{k} \geq 0, \sum_{k=1}^{\infty} c_{k}<\infty, f_{1} \in \operatorname{Id}\left(\mathscr{S}_{W}\right)$.

Theorem 5 is an analogue of the well-known Lévy-Khinchin formula [9, page 9] of the characteristic function of an infinitely divisible probability measure on $\mathbb{R}$. 
THEOREM 6. The class $\tilde{I}_{0}\left(\mathscr{S}_{W}\right)$ consists of all functions $f$ representable in the form

$$
f=\alpha w_{l}+(1-\alpha) w_{k}, \quad \alpha \in[0,1], \quad l, k \in \mathbb{N}_{0} .
$$

COROLlaRY 1. $\operatorname{Id}\left(\mathscr{S}_{W}\right) \cap \tilde{I}_{0}\left(\mathscr{S}_{W}\right)=\left\{0.5 w_{l}+0.5 w_{k}: l, k \in \mathbb{N}_{0}\right\}$

THEOREM 7. $I_{0}\left(\mathscr{S}_{W}\right)=\left\{\alpha w_{l}+(1-\alpha) w_{k}: \alpha \in[0,0.5) \cup(0.5,1], l, k \in \mathbb{N}_{0}\right\}$

$$
=\left\{w_{i} \exp \left(c\left(w_{j}-1\right)\right): c \geq 0, i, j \in \mathbb{N}_{0}\right\} .
$$

It should be mentioned that in the semigroups studied earlier and different from $\mathscr{P}$, the class $I_{0}$ is rather small. Theorem 7 shows that $I_{0}\left(\mathscr{S}_{W}\right)$ is rather large.

The following theorem gives a test for the membership of $N\left(\mathscr{S}_{W}\right)$.

THEOREM 8. Let

$$
f=\sum_{s=0}^{n} \sum_{j=1}^{2^{s-1}} a_{s, j} \psi_{s}^{j}+a_{m, i} \psi_{m}^{i}=\varphi+a_{m, i} \psi_{m}^{i},
$$

where $n<m, a_{m, i}>0$ and the sum $\varphi(t)$ contains at least two non-zero terms. Then $f \in N\left(\mathscr{S}_{\mathbb{W}}\right)$.

\section{Probabilistic interpretation of the semigroup $\mathscr{S}_{W}$}

The semigroup $\mathscr{S}_{\mathscr{W}}$ has an interesting probabilistic interpretation. We first introduce (following [1, Section 14.1]) some notation and definitions.

Let us write $\mathscr{C}$ for the set $\{-1,1\}^{\mathbb{N}}$ of all mappings $\omega: \mathbb{N} \rightarrow\{-1,1\}$. (In [1] $\mathscr{C}$ is defined as the set of all mappings from $\mathbb{Z}$ to $\{-1,1\}$. It is more convenient for us to consider $\mathbb{N}$ instead of $\mathbb{Z}$.) The set $\mathscr{C}$ is a compact abelian group with respect to the pointwise multiplication and the usual product topology. Every element of $\mathscr{C}$ is inverse to itself. We refer to $\mathscr{C}$ as the Cantor-Walsh group.

Let us describe the set $\mathscr{C}^{*}$ of all characters of $\mathscr{C}$. It is easy to see that for all $n \in \mathbb{N}$ the mapping $\rho_{n}: \mathscr{C} \rightarrow\{-1,1\}$ defined as

$$
\rho_{n}(\omega)=\omega(n)
$$

is a character of the group $\mathscr{C}$, called the $n$th Rademacher character. It is proved in [1, Section 14.1.3], that $\mathscr{C}^{*}$ is the set of all finite products of Rademacher characters and the function identically equal to 1 . The set $\mathscr{C}^{*}$ with the discrete topology is a (topological) abelian group with respect to multiplication. Let us denote by $\zeta_{k}(\omega)$, $k \in \mathbb{N}_{0}$, the elements of $\mathscr{C}^{*}$. Every function $\zeta_{k}(\omega)$ is defined by $\rho_{i}(\omega)$ in the same 
manner as $w_{k}(t)$ are defined by $r_{i}(t)$ in (1). The groups $\mathscr{C}^{*}$ and $\mathscr{W}$ are isomorphic via the bijection:

$$
\rho_{n}(\omega) \leftrightarrow r_{n}(t), \quad \zeta_{k}(\omega) \leftrightarrow w_{k}(t)
$$

Let $\mathscr{C}^{* *}$ be the group of all characters of $\mathscr{C}^{*}$. The duality theorem of Pontryagin ([3, Section 24]) implies that $\mathscr{C}^{* *}$ and $\mathscr{C}$ are isomorphic. Note that for every fixed $k \in \mathbb{N}_{0}$, $\zeta_{k}(\omega)$ as a function of $\omega \in \mathscr{C}$ is an element of $\mathscr{C}^{*}$; and for every fixed $\omega \in \mathscr{C}, \zeta_{k}(\omega)$ as a function of $k$ is an element of $\mathscr{C}^{* *} \simeq \mathscr{C}$.

Let us denote by $M^{1}\left(\mathscr{C}^{*}\right)$ the topological semigroup (with operation of convolution and topology of weak convergence) of probability measures on $\mathscr{C}^{*}$. We now recall the general definition of characteristic function. Let $X$ be a second countable locally compact abelian group, $Y$ its group of characters, and let $(x, y)$ stand for the value of $y \in Y$ at $x \in X$. Then the characteristic function $\hat{\mu}$ of the probability measure $\mu$ on $X$ is defined as follows:

$$
\hat{\mu}(y)=\int_{X}(x, y) \mu(d x) .
$$

We apply this definition to the group $X=\mathscr{C}^{*}$. Since $\mathscr{C}^{*}$ is countable, the characteristic function $\hat{\mu}(\omega)$ of the measure $\mu \in M^{1}\left(\mathscr{C}^{*}\right)$ is given by

$$
\hat{\mu}(\omega)=\sum_{k=0}^{\infty} a_{k} \zeta_{k}(\omega), \quad a_{k}=\mu\left(\left\{\zeta_{k}\right\}\right) \geq 0, \quad \sum_{k=0}^{\infty} a_{k}=1
$$

Let us denote by $\hat{M}^{1}\left(\mathscr{C}^{*}\right)$ the multiplicative semigroup of characteristic functions of all measures of $M^{1}\left(\mathscr{C}^{*}\right)$ with the topology of uniform convergence.

Since $\mathscr{C}^{*}$ and $\mathscr{W}$ are isomorphic, we see that the semigroups $\hat{M}^{1}\left(\mathscr{C}^{*}\right)$ and $\mathscr{S}_{\mathscr{W}}$ are isomorphic. Since $M^{1}\left(\mathscr{C}^{*}\right)$ and $\hat{M}^{1}\left(\mathscr{C}^{*}\right)$ are isomorphic, we infer that

$\mathscr{S}_{W}$ is isomorphic to the semigroup $M^{1}\left(\mathscr{C}^{*}\right)$ of probability measures on the group of characters of the Cantor-Walsh group.

Therefore, we can study the arithmetic of $M^{1}\left(\mathscr{C}^{*}\right)$ or $\hat{M}^{1}\left(\mathscr{C}^{*}\right)$. In what follows we use facts related to the arithmetic of probability measures on groups as detailed in [2].

\section{Infinitely divisible and idempotent elements. Proof of Theorem 4 and Theorem 5}

Let us prove Theorem 4. It is known ([2, Section 2.14]) that the set of all idempotent measures on a locally compact abelian group $X$ coincides with the set of shifts of Haar distributions on compact subgroups $K$ of $X$. The characteristic function $\hat{m}_{K}(y)$ of the 
Haar measure $m_{K}$ is given by

$$
\hat{m}_{K}(y)= \begin{cases}1 & y \in A(Y, K) ; \\ 0 & \text { otherwise, }\end{cases}
$$

where $A(Y, K)=\{y \in Y:(x, y)=1$ for all $x \in K\}$. Therefore, the characteristic functions of idempotent measures have the form $\left(x_{0}, y\right) \hat{m}_{K}(y)$, where $x_{0} \in X$. In the case $X=\mathscr{C}^{*}$ we see that the characteristic functions of idempotent elements in $M^{1}\left(\mathscr{C}^{*}\right)$ have the form $\zeta_{j}(\omega) \hat{m}_{K}(\omega)$, where $j \in \mathbb{N}_{0}, K$ is an arbitrary compact subgroup of $\mathscr{C}$, and

$$
\hat{m}_{K}(\omega)= \begin{cases}1 & \text { if } \omega \in A\left(\mathscr{C}^{*}, K\right) \\ 0 & \text { otherwise. }\end{cases}
$$

Since the groups $\mathscr{S}_{W}$ and $\hat{M}^{1}\left(\mathscr{C}^{*}\right)$ are isomorphic, every element of the class $\operatorname{Id}\left(\mathscr{S}_{W}\right)$ has the form $f(t)=w_{j}(t) \tau_{K}(t)$, where $j \in \mathbb{N}_{0}, K$ is an arbitrary compact subgroup of $\mathscr{W}$, and

$$
\tau_{K}(t)= \begin{cases}1 & \text { if } w_{i}(t)=1 \text { for all } w_{i} \in K \\ 0 & \text { otherwise }\end{cases}
$$

It is easy to see that the function $\tau_{K}(t)$ can be represented in the form

$$
\tau_{K}=\prod_{w_{i} \in K}\left(0.5+0.5 w_{i}\right) .
$$

To complete the proof we observe that the compact subgroups of the discrete group $\mathscr{W}$ are exactly all finite subgroups of $\mathscr{W}$.

For the proof of Theorem 5 we need the following theorem which was proved by Parthasarathy, Rao, Varadhan, Sazonov, and which gives the form of the characteristic function of an infinitely divisible measure on a group $X$.

THEOREM 9 ([12], see also [2, Theorem 2.21]). The characteristic function $\hat{\mu}(y)$ of an infinitely divisible measure $\mu$ on $X$ can be represented in the form

$$
\hat{\mu}(y)=\left(x_{0}, y\right) \hat{m}_{K}(y) \exp \left(\int_{X \backslash\{0\}}((x, y)-1-i g(x, y)) \Phi(d x)-\varphi(y)\right),
$$

where $x_{0} \in X, m_{K}$ is the Haar measure of a compact subgroup $K$ of $X, \Phi$ is a measure on $X$ such that $\Phi(X \backslash V)<\infty$ for all neighbourhoods $V$ of zero of $X$, and for all $y \in Y$

$$
\int_{X \backslash\{0\}}(1-\Re(x, y)) \Phi(d x)<\infty
$$


$\varphi(y)$ is a continuous nonnegative quadratic form on $Y$, that is, a continuous nonnegative function on $Y$ such that

$$
\varphi\left(y_{1}+y_{2}\right)+\varphi\left(y_{1}-y_{2}\right)=2\left(\varphi\left(y_{1}\right)+\varphi\left(y_{2}\right)\right) \text { for all } y_{1}, y_{2} \in Y,
$$

$g(x, y)$ is a function on $X \times Y(g$ is independent of $\mu)$ such that the following conditions are valid:

(a) $g(x, y)$ is continuous with respect to the variables $x$ and $y$;

(b) $\sup _{x \in X} \sup _{y \in A}|g(x, y)|<\infty$ for all compact sets $A \subset Y$;

(c) $g\left(x, y_{1}+y_{2}\right)=g\left(x, y_{1}\right)+g\left(x, y_{2}\right)$ for all $x \in X$ and $y_{1}, y_{2} \in Y, g(-x, y)=$ $-g(x, y)$ for all $x \in X, y \in Y$;

(d) for every compact subset $A$ of the group $Y$ there exists a neighbourhood $V_{A}$ of the zero element of $X$ such that $(x, y)=\exp (i g(x, y))$ for all $x \in V_{A}$ and $y \in A$;

(e) for every compact subset $A$ of $Y, g(x, y) \rightarrow 0$ as $x \rightarrow 0 \in X$ uniformly with respect to $y \in A$.

REMARK 1. If every element of $X$ is inverse to itself, then $g(x, y) \equiv 0$. Indeed, if $-x=x$ for all $x \in X$, then the second condition in (c) gives $g(x, y)=-g(x, y)$.

REMARK 2. If every element of $Y$ is inverse to itself, then $\varphi(y) \equiv 0$. Taking $y_{1}=y_{2}=0$ in (5) we have $\varphi(0)=0$. Taking $y_{1}=y_{2}=y$ in (5) we have $\varphi(y+y)+\varphi(0)=4 \varphi(y)$. Since $y=-y$ we conclude that $4 \varphi(y)=2 \varphi(0)=0$.

We apply Theorem 9 to the case $X=\mathscr{C}^{*}$. It is easy to see that $g(x, y) \equiv 0$ satisfies (a)-(e). Indeed, (d) follows if we take for $V_{A}$ the set consisting of one function $\zeta_{0}(\omega) \equiv 1$. This set is open since the topology is discrete, and the other conditions are trivial. By Remark 1 the function $g(x, y) \equiv 0$ is unique. Since $Y=\mathscr{C}^{* *}$ is isomorphic to $\mathscr{C}$ and using Remark 2 , we see that (5) is valid only for the $\varphi(y) \equiv 0$. We note now that if $\Phi$ is a measure on $\mathscr{C}^{*}$ and $\Phi\left(\left\{\zeta_{k}\right\}\right)=c_{k}, k \in \mathbb{N}_{0}$, then the condition $\Phi(X \backslash V)<\infty$ for every neighbourhood $V$ of zero holds if and only if $\sum_{k=1}^{\infty} c_{k}<\infty$. The latter condition implies (4).

Therefore, the characteristic function $\hat{\mu}$ of an infinitely divisible measure $\mu \in$ $M^{1}\left(\mathscr{C}^{*}\right)$ has the form

$$
\hat{\mu}(\omega)=\hat{\mu}_{1}(\omega) \exp \left(\sum_{k=1}^{\infty} c_{k}\left(\zeta_{k}(\omega)-1\right)\right),
$$

where $c_{k} \geq 0, \sum_{k=1}^{\infty} c_{k}<\infty$ and $\hat{\mu}_{1}(\omega)$ is the characteristic function of an idempotent measure $\mu_{1} \in M^{1}\left(\mathscr{C}^{*}\right)$. Since $M^{1}\left(\mathscr{C}^{*}\right)$ and $\mathscr{S}_{\mathbb{W}}$ are isomorphic, we obtain Theorem 5. 


\section{Functions without indecomposable factors. Proof of Theorem 6 and Theorem 7}

We need some lemmas.

LEMMA 1. For all $i \in \mathbb{N}$ and $c \in \mathbb{R}$,

$$
\exp \left(c\left(w_{i}-1\right)\right)=\alpha+\beta w_{i}
$$

where $\alpha=e^{-c} \cosh c, \beta=e^{-c} \sinh c$.

PROOF. Since $w_{i}^{2}(t) \equiv 1$, we have

$$
\begin{aligned}
\exp \left(c\left(w_{i}-1\right)\right) & =e^{-c} \sum_{m=0}^{\infty} \frac{c^{2 m}}{(2 m) !}+e^{-c} \sum_{m=0}^{\infty} \frac{c^{2 m+1}}{(2 m+1) !} w_{i} \\
& =e^{-c} \cosh c+e^{-c} \sinh c w_{i}=\alpha+\beta w_{i}
\end{aligned}
$$

LEMMA 2. Let

$$
\varphi:=\exp \left(c_{i}\left(w_{i}-1\right)+c_{j}\left(w_{j}-1\right)-\varepsilon\left(w_{i} w_{j}-1\right)\right), \quad c_{i}, c_{j}, \varepsilon>0
$$

Then there exists $\varepsilon_{0}=\varepsilon_{0}\left(c_{i}, c_{j}\right)$ such that $\varphi \in \mathscr{S}_{\mathbb{W}}$ for all $\varepsilon \in\left(0, \varepsilon_{0}\right)$.

ProOF. By Lemma 1

$$
\varphi=\left(\alpha+\beta w_{i}\right)\left(\gamma+\delta w_{j}\right)\left(\nu-\sigma w_{i} w_{j}\right)
$$

where $\alpha, \beta, \gamma, \delta, \nu=e^{\varepsilon} \cosh \varepsilon, \sigma=e^{\varepsilon} \sinh \varepsilon>0$, and hence

$$
\begin{aligned}
\varphi= & (\alpha \gamma v-\beta \delta \sigma)+(\beta \gamma v-\alpha \delta \sigma) w_{i} \\
& +(\alpha \delta v-\beta \gamma \sigma) w_{j}+(\beta \delta v-\alpha \gamma \sigma) w_{i} w_{j}
\end{aligned}
$$

Taking $\varepsilon_{0}=\varepsilon_{0}\left(c_{i}, c_{j}\right)$ so small that all coefficients in parentheses in the last formula are positive for $0<\varepsilon<\varepsilon_{0}$, we have $\varphi \in \mathscr{S}_{\mathscr{W}}$.

LEMMA 3. Let

$$
\lambda:=\exp \left(c_{i}\left(w_{i}-1\right)+c_{j}\left(w_{j}-1\right)\right), \quad c_{i}, c_{j}>0, i, j \in \mathbb{N}, i \neq j
$$

Then $\lambda \notin \tilde{I}_{0}\left(\mathscr{S}_{W}\right)$. 
PROOF. Let us write $\lambda=\varphi \psi$, where

$$
\begin{aligned}
\varphi & =\exp \left(c_{i}\left(w_{i}-1\right)+c_{j}\left(w_{j}-1\right)-\varepsilon\left(w_{i} w_{j}-1\right)\right), \\
\psi & =\exp \left(\varepsilon\left(w_{i} w_{j}-1\right)\right), \quad \varepsilon>0 .
\end{aligned}
$$

Since $w_{i} w_{j} \in \mathscr{W}$ and applying Theorem 5 , we have $\psi \in I\left(\mathscr{S}_{W}\right)$. So, $\psi \in \mathscr{S}_{W}$. According to Lemma 2, $\varphi \in \mathscr{S}_{\mathscr{W}}$ for $\varepsilon>0$ small enough. Therefore, $\varphi$ is a factor of $\lambda$. Let us prove that $\varphi \notin I\left(\mathscr{S}_{W}\right)$. If this is not the case, then $\varphi$ can be represented as

$$
\exp \left(\sum_{i=1}^{\infty} c_{i}\left(w_{i}-1\right)\right), \quad c_{i} \geq 0, \quad \sum_{i=1}^{\infty} c_{i}<\infty .
$$

This is a consequence of positivity of $\varphi$, Theorem 4 and Theorem 5. But $w_{i} w_{j}=w_{k}$ for some $k \neq 0, i, j$, and we have a contradiction to the uniqueness of the decomposition as a series of the Walsh functions. By Theorem $2, \varphi \notin \tilde{I}_{0}\left(\mathscr{S}_{\mathscr{W}}\right)$, and hence $\lambda \notin$ $\tilde{I}_{0}\left(\mathscr{S}_{W}\right)$.

LEMMA 4. Let

$$
\xi:=\left(0.5+0.5 w_{i}\right)\left(0.5+0.5 w_{j}\right), \quad i, j \in \mathbb{N}, \quad i \neq j .
$$

Then $\xi \notin \tilde{I}_{0}\left(\mathscr{S}_{\mathscr{W}}\right)$.

PROOF. We note that $\xi=1$ for $w_{i}=1, w_{j}=1$ and $\xi=0$ otherwise. We also note that the function $\lambda$ in Lemma 3 satisfies the condition: $\lambda=1$ for $w_{i}=1, w_{j}=1$. Therefore, $\xi=\xi \lambda$. Lemma 4 follows now from Lemma 3 .

LEMMA 5. Let

$$
\eta:=\left(0.5+0.5 w_{i}\right) \exp \left(c_{j}\left(w_{j}-1\right)\right), \quad c_{j}>0, \quad i, j \in \mathbb{N}, \quad i \neq j .
$$

Then $\eta \notin \tilde{I}_{0}\left(\mathscr{S}_{W}\right)$.

ProOF. We have

$$
0.5+0.5 w_{i}=\left(0.5+0.5 w_{i}\right) \exp \left(c_{i}\left(w_{i}-1\right)\right)
$$

for all $c_{i}>0$. To prove this identity, it is sufficient to substitute +1 and -1 for $w_{i}$. Therefore, we can write $\eta$ in the form

$$
\eta=\left(0.5+0.5 w_{i}\right) \lambda,
$$

where $\lambda$ is the function of Lemma 3. Lemma 5 follows now from Lemma 3. 
LEMMA 6. Let

$$
\zeta:=\alpha w_{m}+(1-\alpha) w_{k}
$$

where $0 \leq \alpha \leq 1, m, k \in \mathbb{N}_{0}, m \neq k$. Then $\zeta \in \tilde{I}_{0}\left(\mathscr{S}_{\mathscr{W}}\right)$. In addition,

(1) if $\alpha \neq 0.5$, then $\zeta \in I_{0}\left(\mathscr{S}_{W}\right)$;

(2) if $\alpha=0.5$, then $\zeta \in \tilde{I}_{0}\left(\mathscr{S}_{W}\right) \backslash I_{0}\left(\mathscr{S}_{W}\right)$.

PROOF. The case $\alpha=0$ or $\alpha=1$ is trivial. Let us consider $0<\alpha<1$. Let $\zeta=\zeta_{1} \zeta_{2}$, where $\zeta_{1}, \zeta_{2} \in \mathscr{S}_{\mathbb{W}}, \zeta_{1} \neq w_{k}$. First we note that the expansion of $\zeta_{1}$ into the series of the Walsh functions contains exactly two nonzero terms. Indeed, if there are three or more terms, then the series expansion of $\zeta$ also contains three or more terms. This gives a contradiction. We have used here that if $i \neq j$, then $w_{i} w_{k} \neq w_{j} w_{k}$. We have proved that

$$
\zeta_{1}=a w_{p}+(1-a) w_{q}, \quad 0<a<1, \quad p \neq q .
$$

Later on we use the following fact. A convex linear combination of two distinct Walsh functions is equal to zero at some point if and only if the coefficients of this linear combination are both 0.5 .

(1) Assume $\alpha \neq 0.5$. Then we have $\zeta(t) \neq 0$ for all $t \in[0,1]$. Therefore, $\zeta_{1}(t) \neq 0$, and hence $a \neq 0.5$. We can assume without loss of generality that $a>0.5$. By Lemma 1 we conclude that

$$
\begin{aligned}
\zeta_{1} & =w_{p}\left(a+(1-a) w_{p} w_{q}\right) \\
& =w_{p}\left(a+(1-a) w_{i}\right)=w_{p} \exp \left(c\left(w_{i}-1\right)\right), \quad c>0 .
\end{aligned}
$$

By Theorem 5, $\zeta_{1} \in I\left(\mathscr{S}_{W}\right)$. Therefore, $\zeta_{1}$ is decomposable. Since $\zeta_{1}(t) \neq 0$ for $t \in[0,1], \zeta_{1}$ is not a non-degenerate idempotent function, and hence $\zeta \in I_{0}\left(\mathscr{S}_{W}\right)$.

(2) Assume $\alpha=0.5$. Then the function $\zeta$ has zeros on $[0,1]$ and the coefficient $a$ in the definition of $\zeta_{1}$ can be equal to 0.5 . In this case also the function $\zeta_{1}=0.5 w_{p}+0.5 w_{q}$ is decomposable. This follows from the representation

$$
\zeta_{1}=0.5 w_{p}+0.5 w_{q}=w_{p}\left(0.5+0.5 w_{i}\right)=w_{p}\left(0.5+0.5 w_{i}\right)^{2} .
$$

Therefore, $\zeta \in \tilde{I}_{0}\left(\mathscr{S}_{W}\right)$. But $\zeta$ is a non-degenerate idempotent element for $\alpha=0.5$, and hence $\zeta \notin I_{0}\left(\mathscr{S}_{\mathscr{W}}\right)$.

Proof (of Theorem 6 and Theorem 7). Let $f \in \tilde{I}_{0}\left(\mathscr{S}_{W}\right)$. According to Theorem 2, Theorem 4 and Theorem 5 we have

$$
f=w_{j} \prod_{w_{i} \in K}\left(0.5+0.5 w_{i}\right) \exp \left(\sum_{k=1}^{\infty} c_{k}\left(w_{k}-1\right)\right),
$$


where $j \in \mathbb{N}_{0}, c_{k} \geq 0, \sum_{k=1}^{\infty} c_{k}<\infty$, and $K$ is a finite subgroup of $W$. It follows from Lemma 3 that only one coefficient $c_{k}$ can be non-zero, for otherwise $f$ has an indecomposable factor. Lemma 4 implies that either $K=\left\{w_{0}\right\}$ or $K=\left\{w_{0}, w_{i}\right\}$, for otherwise $f$ has an indecomposable factor. It follows from Lemma 5 that the case $K=\left\{w_{0}, w_{i}\right\}$ is possible only if $c_{k}=0$ for all $k \in \mathbb{N}$, for otherwise $f$ has an indecomposable factor. Therefore, if $f \in \tilde{I}_{0}\left(\mathscr{S}_{W}\right)$, then either $f=w_{j}\left(0.5+0.5 w_{i}\right)$ or $f=w_{j} \exp \left(c_{i}\left(w_{i}-1\right)\right)$. According to Lemma $1 f=\alpha w_{m}+(1-\alpha) w_{k}$ where $0 \leq \alpha \leq 1, m, k \in \mathbb{N}_{0}, m \neq k$. Theorem 6 and Theorem 7 now follow from Lemma 6.

REMARK 3. Theorem 7 can also be deduced from known general theorems on decomposition of the generalized Poisson distribution on groups. Let $E_{x}$ be the probability measure on $X$ concentrated at the point $x \in X$. For every measure $\Phi$ on $X$ the generalized Poisson distribution is defined by the formula

$$
e(\Phi):=\exp (-\Phi(X))\left(E_{0}+\Phi+\frac{\Phi^{2 *}}{2 !}+\cdots+\frac{\Phi^{n *}}{n !}+\cdots\right) .
$$

THEOREM 10 (Rukhin, see [2, Section 6, Proposition 6.6]). Let $\mu=e(\Phi)$, where $\Phi=\psi E_{x}, \psi>0, x \in X$.

Then $\mu \in I_{0}(M(X))$ if $x$ is either element of infinite order or order 2 and $\mu \notin$ $I_{0}(M(X))$ if $x$ is element of order $p>2$.

THEOREM 11 (Fel'dman, see [2, Section 6, Proposition 6.11]). Let $\Phi=\psi_{1} E_{x_{1}}+$ $\psi_{2} E_{x_{2}}$, where $\psi_{j}>0, x_{j} \neq 0,2 x_{j}=0, j=1,2, x_{1} \neq x_{2}$. Then $\mu=e(\Phi) \notin$ $I_{0}(M(X))$.

Since all elements of $\mathscr{C}^{*}$, except zero, have order 2 it follows from Theorem 10 that

$$
\exp \left(c\left(w_{i}-1\right)\right) \in I_{0}\left(\mathscr{S}_{\mathbb{W}}\right), \quad i \in \mathbb{N}, \quad c \geq 0 .
$$

It follows from Theorem 11 that

$$
\exp \left(c_{i}\left(w_{i}-1\right)+c_{j}\left(w_{j}-1\right)\right) \notin I_{0}\left(\mathscr{S}_{\mathscr{W}}\right), \quad c_{i}, c_{j}>0, \quad i \neq j, \quad i, j \in \mathbb{N} .
$$

\section{Indecomposable elements of $\mathscr{S}_{W}$. Proof of Theorem 8}

We use the following notation.

$$
\begin{aligned}
& W_{m}:=\left\{\psi_{m}^{i}\right\}_{i=1}^{m-1} \text { is the set of Walsh functions of the } m \text { th series, } \\
& W_{(<m)}:=\bigcup_{j=0}^{m-1} W_{j} ; \quad W_{(>m)}:=\bigcup_{j=m+1}^{\infty} W_{j} .
\end{aligned}
$$

The definition of the Walsh functions implies the following statement. 
REMARK 4. If $w_{i}, w_{j} \in W_{m}$, then $w_{i} w_{j} \in W_{(<m)}$. If $w_{i} \in W_{m}, w_{j} \in W_{k}$, and $k<m$ then $w_{i} w_{j} \in W_{m}$.

PROOF (of Theorem 8). Let $f$ has the form (3). Assuming $f \notin N\left(\mathscr{S}_{W}\right)$ we have $f=f_{1} f_{2}$, where $f_{1}, f_{2} \in \mathscr{S}_{\mathscr{W}}, f_{1}, f_{2} \neq w_{k}, k \in \mathbb{N}_{0}$. Without loss of generality we may assume that the coefficient $c_{0,1}$ of the function $\psi_{0}^{1}(t) \equiv 1$ in the series expansion of $f_{2}$ is non-zero. We divide the proof of Theorem 8 into several steps.

(a) We note that $f_{1}$ does not contain any term from $W_{(>m)}$, for otherwise condition $c_{0,1} \neq 0$ implies that $f$ contains terms from $W_{(>m)}$. This is a contradiction.

(b) $f_{1}$ contains exactly one term from $W_{m}$, namely $\psi_{m}^{i}$. If there are two such terms, then the condition $c_{0,1} \neq 0$ implies that $f$ contains two terms from $W_{m}$. This is a contradiction. If $f_{1}$ does not contain any term from $W_{m}$, then it contains at least two terms from $W_{(<m)}$. Hence, according to the condition $a_{m, i}>0, f_{2}$ contains at least one term from $W_{m}$ (see Remark 4). Then $f$ contains at least two terms from $W_{m}$. This is a contradiction.

(c) $f_{1}$ contains at least one term from $W_{(<m)}$ because $f_{1}$ contains at least two terms in general.

(d) $f_{2}$ does not contain any term from $W_{(>m)}$. Indeed, if there is such a term, then it is contained also in $f$ (see (c) and Remark 4).

(e) $f_{2}$ contains exactly one term from $W_{m}$. If there are two such terms then according to (c) $f$ contains two such terms, but this is not the case. If there is no such term, then $f_{2}$ contains at least two terms from $W_{(<m)}$. Consequently, according to $(\mathrm{b}), f$ contains two terms from $W_{m}$, which is not the case.

(f) $f_{2}$ contains exactly one term from $W_{(<m)}$, namely $\psi_{0}^{1} \equiv 1$. If there are two such terms, then according to (b) $f$ has two terms from $W_{m}$.

(g) $f_{1}$ has exactly one term from $W_{(<m)}$. If there are two such terms, then according to (e) $f$ has two terms from $W_{m}$. This is not the case.

It follows from (a)-(g) that

$$
f_{1}=a \psi_{n}^{k}+b \psi_{m}^{i}, n<m, \quad \text { and } \quad f_{2}=c+d \psi_{m}^{l}
$$

Then

$$
f=a c \psi_{n}^{k}+b c \psi_{m}^{i}+a d \psi_{n}^{k} \psi_{m}^{l}+b d \psi_{m}^{i} \psi_{m}^{l}
$$

Since $f$ has only one term from $W_{m}$ and since $\psi_{n}^{k} \psi_{m}^{l} \in W_{m}$ (Remark 4), we have $\psi_{n}^{k}=\psi_{0}^{1} \equiv 1$ and $l=i$. Therefore,

$$
f=(a c+b d)+(b c+a d) \psi_{m}^{i},
$$

that is, $f$ contains exactly two terms. This is a contradiction. 
We now present a sequence of functions from $N\left(\mathscr{S}_{W}\right)$ which is uniformly convergent to a given function $f \in \mathscr{S}_{W}$. Let

$$
f=\sum_{k} \sum_{j} a_{k, j} \psi_{k}^{j}
$$

We consider two cases: (i) there are at least two terms in $f$, (ii) $f=\psi_{k}^{j}$.

In the first case we take

$$
f_{n}=\sum_{k=0}^{n} \sum_{j} \frac{a_{k, j}}{S_{n}} \psi_{k}^{j}+\frac{1}{n} \psi_{n+1}^{1} \quad\left(S_{n}=\frac{n}{n-1} \sum_{k=0}^{n} \sum_{j} a_{k, j}\right) .
$$

Let $n$ be so large that the sum in the definition of $f_{n}$ contains at least two terms. According to Theorem $8, f_{n} \in N\left(\mathscr{S}_{W}\right)$. It is evident that $f_{n} \rightarrow f$ as $n \rightarrow \infty$ uniformly on $[0,1]$.

In the second case, we consider

$$
f_{n}=\left(1-\frac{2}{n}\right) \psi_{k}^{j}+\frac{1}{n} \psi_{k+1}^{1}+\frac{1}{n} \psi_{k+2}^{1}
$$

It follows from Theorem 8 that $f_{n} \in N\left(\mathscr{S}_{\mathscr{W}}\right)$. Evidently, $f_{n} \rightarrow f$ as $n \rightarrow \infty$ uniformly.

\section{Acknowledgements}

I wish to express gratitude to Professor I. V. Ostrovskii for posing the problem and his attention to this work and to the referee for helpful comments on a preliminary version of this paper.

\section{References}

[1] R. E. Edwards, Fourier series. A modern introduction, vol. 2 (Springer, New York, 1982).

[2] G. M. Fel'dman, Arithmetic of probability distributions and characterization problems on Abelian groups, Transl. Math. Monographs 116 (Amer. Math. Soc., Providence, R.I., 1993).

[3] E. Hewitt and K. A. Ross, Abstract harmonic analysis, vol. 1 (Springer, Berlin, 1963).

[4] I. P. Il'inskaya (Trukhina), 'Arithmetic of a semigroup of series in Legendre functions of the second kind', Turkish J. Math. 21 (1997), 357-373.

[5] S. Kaczmarz and H. Steinhaus, Theorie der Orthogonalreien ( $\mathrm{Z}$ subwencji funduszu kultury narodowej, Warszawa, 1935).

[6] B. S. Kashin and A. A. Saakyan, Orthogonal series (Nauka, Moscow, 1984) (in Russian).

[7] D. G. Kendall, 'Delphic semigroups, infinitely divisible regenerative phenomena, and the arithmetic of p-functions', Z. Wahrscheinlichkeitstheorie und Verw. Gebiete 9 (1967), 163-195. 
[8] - A tribute to the memory of Rollo Davidson (Willey and Sons, London, 1975).

[9] Ju. V. Linnik and I. V. Ostrovskii, Decomposition of random variables and vectors, Transl. Math. Monographs 48 (Amer. Math. Soc., Providence, R.I., 1977).

[10] I. V. Ostrovskii, 'The arithmetic of probability distributions', Theory Probab. Appl. 31 (1986), $1-24$.

[11] K. R. Parthasarathy, R. R. Rao and S. R. S. Varadhan, 'On the category of indecomposable distributions on topological groups', Trans. Amer. Math. Soc. 102 (1962), 200-217.

[12] - 'Probability distributions on locally compact abelian groups', Illinois J. Math. 7 (1963), 337-369.

[13] I. P. Trukhina, 'On a problem connected with the arithmetic of probability measures on spheres', Zap. Nauch. Sem. Leningrad. Otdel. Mat. Inst. Steklov (LOMI) 87 (1979), 143-158 (in Russian).

[14] _- 'Arithmetic of spherically symmetric measures in the Lobachevskii space', Teor. Funktsil Funktsional. Anal. i Prilozhen. 34 (1980), 136-146 (in Russian).

[15] K. Urbanik, 'Generalized convolutions', Studia Math. 23 (1963), 217-245.

Kharkov State University

Department of Mathematics

4 Svobody Square

310077 Kharkov

Ukraine

e-mail: iljinskii@ilt.kharkov.ua 